\title{
Classification of hypocholesterolemia lipid patterns using Chol/Trig Combination System
}

\author{
KINUE OOI ${ }^{1}$, KAZUSHI SUGIMOTO ${ }^{2}$, KATSUYA SHIRAKI ${ }^{2}$, NORIHIKO YAMAMOTO ${ }^{2}$, MASAHIKO TAMEDA ${ }^{2}$, \\ TETSUYA BEPPU ${ }^{2}$, JUNICHIRO TANAKA ${ }^{2}$, KEIICHIRO NOJIRI ${ }^{2}$, SATOKO KUSAGAWA ${ }^{2}$, \\ YOSHIYUKI TAKEI ${ }^{2}$, CHIAKI MASUDA ${ }^{1}$ and TSUTOMU NOBORI ${ }^{1}$
}

Department of ${ }^{1}$ Laboratory Medicine; ${ }^{2}$ Gastroenterology, Mie University School of Medicine, Tsu, Mie 514-8507, Japan

Received September 21, 2009; Accepted November 6, 2009

DOI: 10.3892/ijmm_00000382

\begin{abstract}
Patterns of hypocholesterolemic lipid fractions in 295 patients with liver diseases, malignant tumors, arteriosclerotic and renal diseases with cholesterol (Chol) levels of $<130 \mathrm{mg} / \mathrm{dl}$ were classified using a simultaneous analytical method for the Chol and triglyceride (TG) fractions (Chol/Trig Combo System). Hypocholesterolemia was classified as follows: IV, Type IV on WHO hyperlipidemia phenotype classification; intermediate density lipoprotein (IDL), cases with appearance of IDL, including appearance of $\mathrm{Lp}(\mathrm{a})$; high + low density lipoproteins (HDL+LDL), lipids mostly consisting of HDL and LDL fractions; HDL abnormality, cases with slow $\alpha$ HDL or fast HDL; abnormal LDL, both Chol and TG fractions mostly consisting of LDL fraction; normal type, ratios of HDL, very low density lipoproteins (VLD) and LDL fractions were almost normal; and low HDL, HDL-C was $<30 \mathrm{mg} / \mathrm{dl}$. Many patients with liver diseases had HDL+LDL (45\%), and abnormal LDL was noted in $13 \%$ of the cases. In malignant tumors, the frequencies of low HDL, normal type, and HDL+LDL cases were similar (22-30\%). In arteriosclerosis, normal type accounted for $46 \%$ of the cases, and the frequency of normal type was higher $(60 \%)$ in renal diseases. Mortality rate (within 1 year after measurement) was then compared among lipid patterns. In liver diseases, mortality rate increased in the following order: abnormal LDL (55\%); low HDL (31\%); HDL abnormality (25\%); and HDL+LDL
\end{abstract}

Correspondence to: Dr Katsuya Shiraki, Department of Gastroenterology, Mie University School of Medicine, 2-174 Edobashi, Tsu, Mie 514-8507, Japan

E-mail: katsuyas@clin.medic.mie-u.ac.jp

Abbreviations: TC, total cholesterol; TG, triglyceride; HDL, highdensity lipoprotein; VLDL, very low-density lipoprotein; LDL, low-density lipoprotein; IDL, intermediate low-density lipoprotein; $\mathrm{CM}$, chylomicron; CMF, charge modification frequency; REP, rapid electrophoresis system; Lp(a), lipoprotein (a); DM, diabetes mellitus; AGE, advanced glycation end products

Key words: hypocholesterolemia, lipid pattern, cholesterol fraction, triglyceride fraction charge modification frequency
(21\%). No deaths were seen among patients with normal type. In malignant tumors, mortality rate was very high $(88 \%)$ in patients with HDL+LDL, but low in patients with normal type (22\%) and low HDL (9\%). Mortality rate was low in patients with arteriosclerosis and renal diseases in the short-term follow-up period (1 year). In the comparisons of distribution, mean, and appearance rate of charge modification frequency $(\mathrm{CMF})$ among lipid patterns, parameters were high in all patterns other than HDL+LDL. Classification of hypocholesterolemia lipid patterns and evaluation of CMF may therefore be clinically useful.

\section{Introduction}

WHO hyperlipidemia phenotyping and investigation of abnormal lipoprotein have become simple methods of simultaneously analyzing cholestrol ( $\mathrm{Chol})$ and triglyceride (TG) fractions (Chol/Trig Combo System) (1), and are widely used in routine examinations. In addition, numerical conversion of low density lipoprotein (LDL) mobility toward the anode (or cathode), known as charge modification frequency (CMF), has become a method of investigating degenerated LDL (2), which is more closely related to arteriosclerosis than other LDL species. Lipid testing is essential for diagnosis and treatment of hyperlipidemia. With regard to the epidemiological relationship between Chol levels and prevalence/mortality rate, ischemic heart disease is more frequent with increases in Chol, while patients with severe diseases, such as cancers, become more common and show a higher mortality rate with decreases in Chol (3-7).

For primary hypocholesterolemia, there have been many detailed reports on gene analysis $(8,9)$. Many cases of secondary hypocholesterolemia have also been reported (10), and its relationship with total cholesterol (TC) levels has been investigated in numerous diseases and pathologies, such as liver diseases, hyperthyroidism, malignant tumors, subnutrition, infectious diseases, anemia, acute myocardial infarction, blood diseases, and drug administration have been reported as causes (11-17). Iseki et al (14) reported that TC ranged from 76 to $378 \mathrm{mg} / \mathrm{dl}$ in dialysis patients, and that mortality rate was significantly higher in patients with hypocholesterolemia, thus confirming that hypocholesterolemia is an independent predictor. However, there have been fewer reports on individual lipid fractions. Chiang et al (11) investigated TC, TG, high 
density lipoprotein (HDL)-C and LDL-C, and found that TC and LDL-C were useful.

In this study, we classified lipid fraction patterns in hypocholesterolemia using a Chol/Trig Combo System, and investigated the possibility of determining the pathology of liver diseases, malignant tumors, arteriosclerotic diseases and renal diseases based on these lipid patterns.

\section{Materials and methods}

Subjects. Subjects were 295 in- and out-patients having hypocholesterolemia with $\mathrm{Chol}<130 \mathrm{mg} / \mathrm{dl}$ at the hospital of Mie University School of Medicine. Diseases included liver diseases, 85 patients; malignant tumors, 37; arteriosclerotic diseases, 68; renal diseases, 74; diabetes; 7; collagen disease, 9; and other diseases in 15 . As controls, 102 healthy subjects and 620 patients with normolipidemia (liver diseases, 86; malignant tumors, 51; arteriosclerotic diseases, 119; renal diseases, 97; diabetes, 97; and collagen disease, 68) were investigated. All liver cancers, including hepatocellular carcinoma, were classified as liver disease.

Methods. Chol and TG fractions were subjected to agarose gel electrophoresis using an automatic REP (Helena Laboratories, Beaumont, TX, USA), followed by fractional staining with CholeTri Combo CH and TG (KK Helena Kenkyujo, Saitama, Japan), respectively, in order to fractionate HDL, very low density lipoprotein (VLDL), IDL, LDL and chylomicron (CM). Migration patterns were analyzed using the lipid analysis software EdBank (KK Helena Kenkyujo, Saitama, Japan). In measurements of CMF, the distance of the normal LDL position from the application site was regarded as $100 \%$, and the mobility of LDL in the sample was presented as an integral percentage change relative to normal LDL.

Hypocholesterolemia was classified as follows: IV, Type IV on WHO hyperlipidemia phenotype classification; IDL, cases with appearance of IDL, including appearance of Lp(a); HDL+LDL, lipids mostly consisted of HDL and LDL fractions with undetectable or very low VLDL; HDL abnormality, abnormality in the HDL fraction, such as fast HDL and slow aHDL, was noted; abnormal LDL, abnormal LDL was noted; low HDL, HDL levels were $<30 \mathrm{mg} / \mathrm{dl}$; and normal type, ratios of HDL, VLDL and LDL fractions were almost normal.

Normal levels of TC and TG are 130-220 and 35-150 mg/dl, respectively. Patients who died within 1 year after measurement were regarded as death cases.

\section{Results}

Classification of hypocholesterolemia. Classifications of hypocholesterolemia, and one example for each pattern are shown in Fig. 1. IV, Type IV on WHO hyperlipidemia phenotype classification; TG levels were high. IDL, appearance of IDL; IDL appeared at a position between VLDL and LDL. Cases with appearance of $L p(a)$ were included in this classification. IDL, appearance of $\operatorname{Lp}(\mathrm{a})$. As $\mathrm{Lp}(\mathrm{a})$ is Chol-rich, these cases were distinguished from cases with appearance of IDL, but because the mobility is subtly different among the phenotypes, some cases could not be distinguished, even though Lp(a) levels were high. HDL+LDL, mostly consisting of HDL and LDL fractions, and VLDL was undetectable or very low. HDL

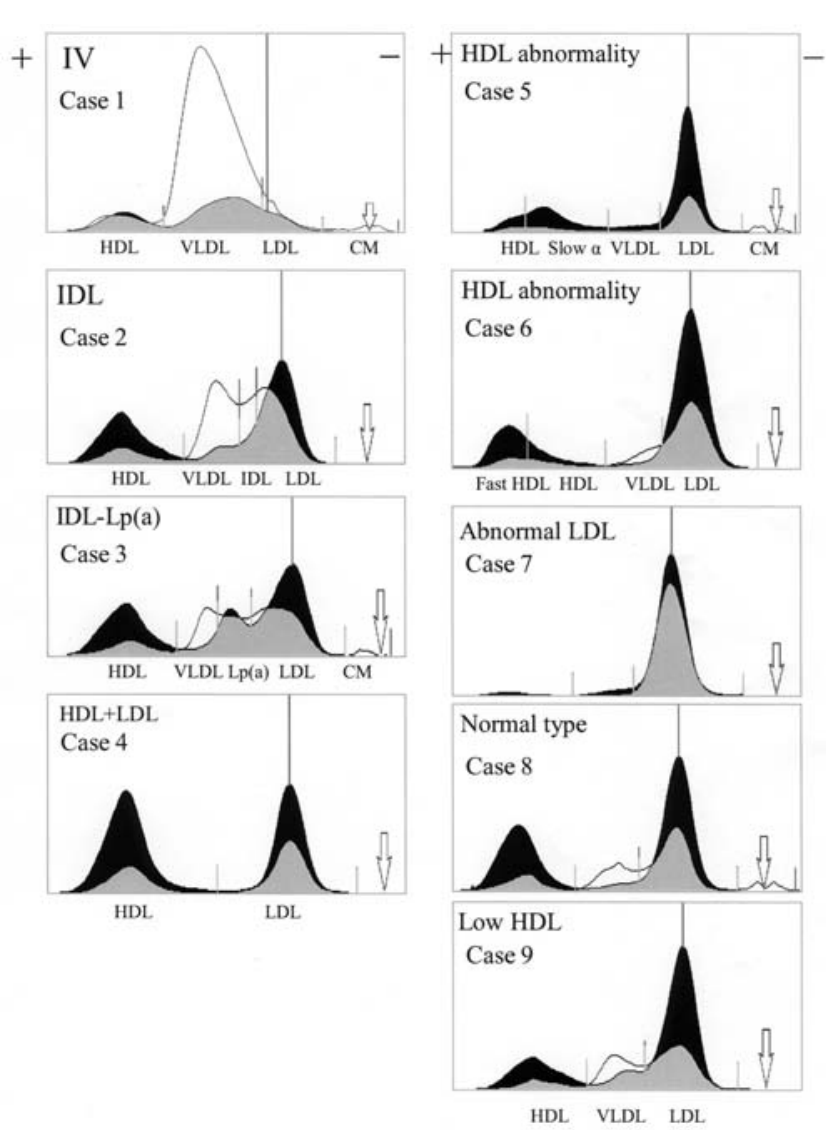

Figure 1. Classification of hypocholesterolemia lipid patterns using Chol/Trig Combo. $\preccurlyeq$, origin; black, cholesterol fraction; white, triglyceride fraction; grey, overlap region; I, patient LDL-C peak line; CMF, charge modification frequency. Case 1, 40-year-old male with familial complex-type hyperlipidemia and renal failure; 2, 71-year-old male with atrial fibrillation and angina pectoris; 3, 63-year-old female with diabetes; 4, 62-year-old female with hepatocellular carcinoma and hepatic cirrhosis who died after 2 years and 2 months; 5, 26-year-old male with fulminant hepatitis and hepatic encephalopathy; 6: 60-year-old male with hepatocellular carcinoma and hepatic cirrhosis; 7, 44-year-old male with hepatocellular carcinoma and hepatic cirrhosis who died after 4 months; 8, 61-year-old male with renal failure under dialysis treatment; 9, 71-year-old male with heart failure and myocardial infarction.

abnormality, slow $\alpha$ HDL. Slow $\alpha$ HDL is likely to appear in mild cholestasis, and often noted in PBC, in which HDL-C and Chol are often high. In other diseases, slow $\alpha \mathrm{HDL}$ is often low and unclear. HDL abnormality, fast HDL. This is usually caused by the influence of free fatty acids. When free fatty acid levels are high, all fractions shift toward the anode, while low free fatty acid levels only affects the HDL fraction. Abnormal LDL, abnormal composition in which both Chol and TG fractions mostly consist of LDL fraction, and HDL and VLDL fractions are not detected, showing that the LDL fraction is both Chol- and TG-rich, in contrast to the normal LDL fraction, in which only Chol is abundant. Normal type, almost normal ratios of HDL, VLDL, and LDL fractions. Low HDL, HDL-C levels of $<30 \mathrm{mg} / \mathrm{dl}$. HDL-C levels $\geq 40 \mathrm{mg} / \mathrm{dl}$ are usually regarded as normal, but levels $<30 \mathrm{mg} / \mathrm{dl}$ are regarded as low HDL, as the patients were hypocholesterolemic.

Distribution of lipid patterns. In the distribution of the lipid patterns in hypocholesterolemia based on the above classification (Fig. 2A), the frequency of HDL+LDL was the highest, accounting for $44.7 \%$ (38/85), in patients with liver 
A

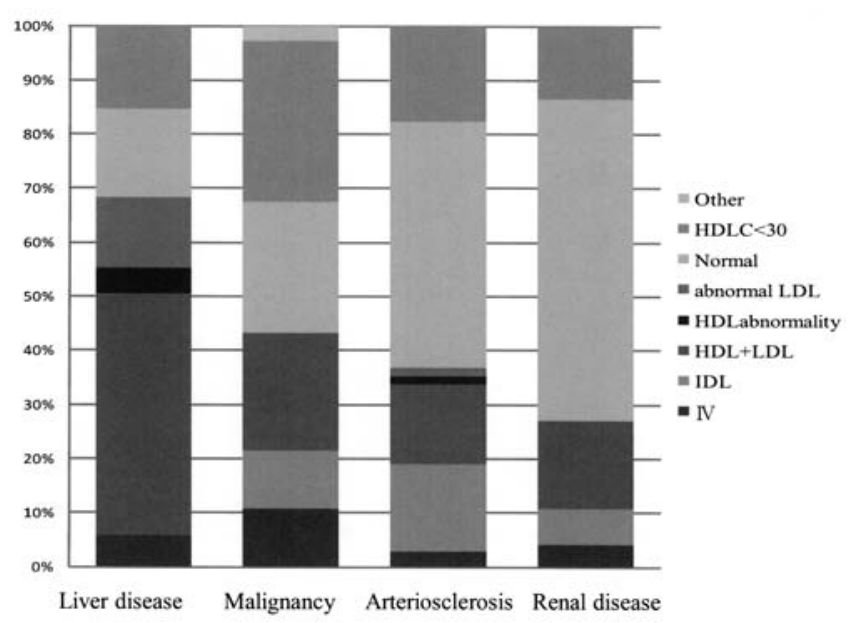

B

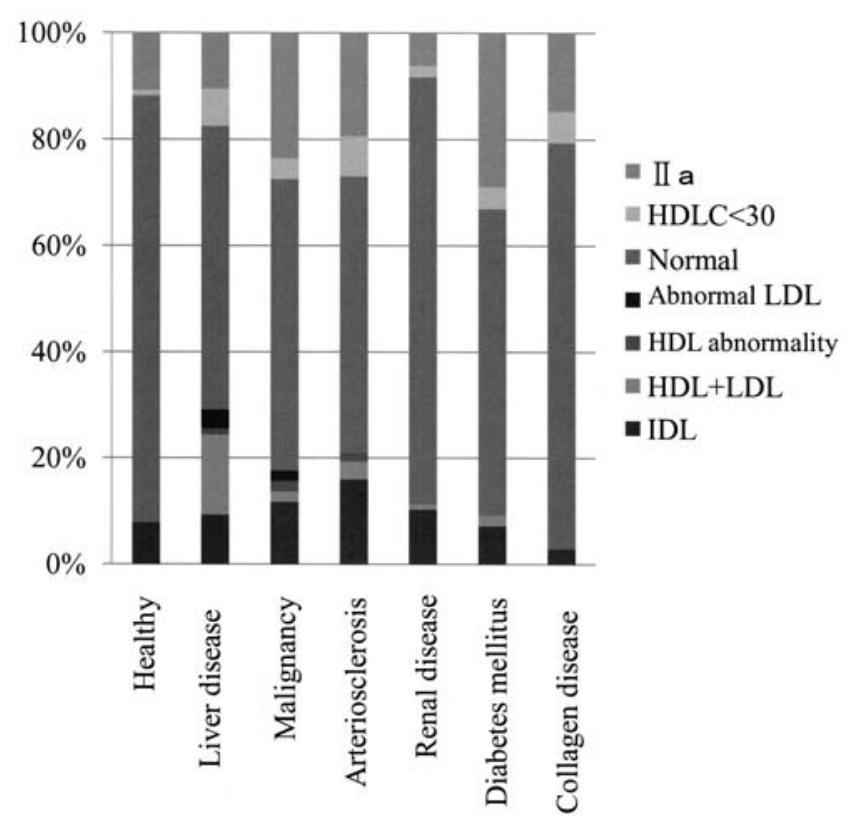

Figure 2. Distribution of lipid patterns. Distribution of lipid patterns in various diseases in hypocholesterolemic (A) and normolipidemic (B) subjects.

diseases, followed by normal type (16.5\%), low HDL (15.3\%) and abnormal LDL (12.9\%). In patients with malignant tumors, the frequency of low HDL was the highest $(29.7 \%)$, followed by normal type $(24.3 \%)$ and HDL+LDL $(21.6 \%)$. In patients with arteriosclerosis, the frequency of normal type was highest (45.6\%), followed by low HDL (17.6\%) and IDL (16.2\%). In patients with renal diseases, the frequency of normal type was the highest $(59.5 \%)$, even though the Chol levels were low. The frequencies of HDL+LDL and low HDL were 16.2 and 13.5\%, respectively.

As a control, lipid pattern distributions were investigated in healthy subjects and normolipidemic patients with various diseases (Fig. 2B). Normal type accounted for more than half of patients in all disease groups (52.1-80.4\%), and the highest frequency was noted in healthy subjects and patients with renal diseases $(80.4 \%)$. When the frequencies of type IIa, in which HDL-C levels are low, and low HDL were combined, the frequency was highest in diabetes $(33.0 \%)$, followed by

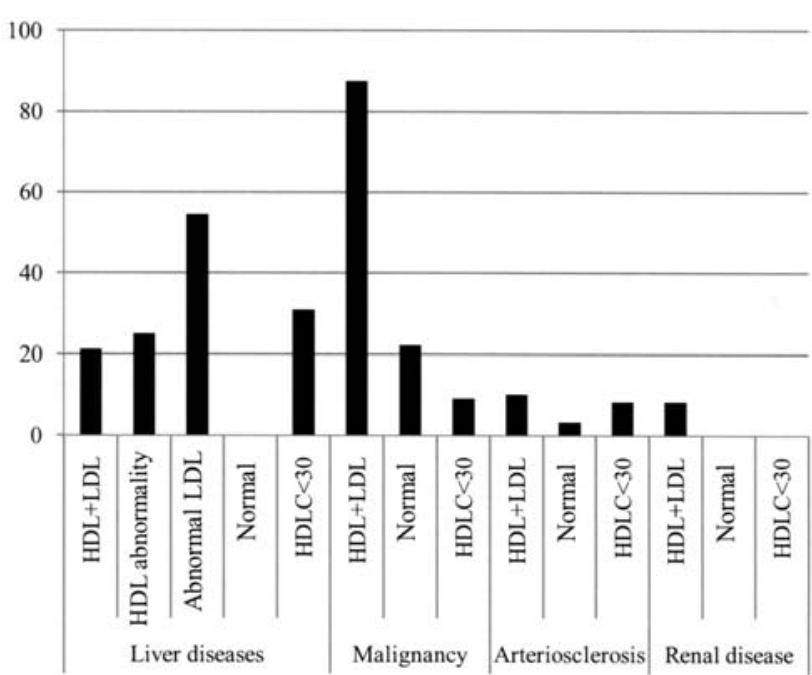

Figure 3. Comparison of mortality rate in relation to lipid patterns. Comparison of mortality rate in subjects with various diseases according to lipid patterns.

malignant tumors $(27.5 \%)$, arteriosclerosis $(26.9 \%)$, collagen disease $(20.6 \%)$, liver diseases $(17.4 \%)$ and healthy subjects (11.8\%). The frequency of IDL was $2.9-16.0 \%$, and it was high in arteriosclerosis (16.0\%), malignant tumors (11.8\%) and renal diseases $(10.3 \%)$. The lowest frequency was noted in collagen disease $(2.9 \%)$. In patients with liver diseases, HDL+LDL and abnormal LDL were seen in 15.1 and $3.5 \%$ of patients, respectively.

Mortality rate in relation to lipid patterns. With regard to patients who died within 1 year after measurement, the mortality rate was compared among lipid patterns (Fig. 3). The mortality rate of hypocholesterolemic patients was low in arteriosclerosis and renal diseases, but high in liver diseases and malignant tumors. The mortality rates of patients with normolipidemia and hypocholesterolemia were 5.7 and $22.3 \%$, respectively, in liver diseases, and 3.9 and $29.7 \%$, respectively, in malignant tumors, thus confirming low mortality rates in normolipidemic patients.

In a comparison between liver diseases and malignant tumors, mortality rates varied depending on lipid patterns. In liver diseases, the highest mortality rate $(54.5 \%)$ was noted in patients with abnormal LDL, followed by low HDL (30.8\%), HDL abnormality (25.0\%), and HDL+LDL (21.1\%), and no deaths were seen among patients with normal type. In malignant tumors, the mortality rate was very high $(87.5 \%)$ in patients with HDL+LDL, and low in patients with normal type (22.2\%) and HDL+LDL (9.2\%). One of the patients with type IV died.

TC levels among the lipid patterns in the 4 disease groups ranged from 97 to $119 \mathrm{mg} / \mathrm{dl}$ in all patterns, and no significant differences were seen, other than the low levels $(79.0 \mathrm{mg} / \mathrm{dl})$ present in patients having liver diseases with low HDL.

Comparison of distribution, mean and appearance rate of CMF in relation to lipid patterns. CMF was $\geq 20$ in $\mathrm{HDL}+\mathrm{LDL}$ and low HDL in liver diseases, IV+IDL and HDL+LDL in malignant tumors, and all patterns (IV+IDL, normal type, and low HDL), excluding HDL+LDL, in renal diseases (Fig. 4A). In normal type, $\mathrm{CMF}$ was not high in many cases in all disease groups, excluding renal diseases. 
A Scatter diagram of charge modification frequency

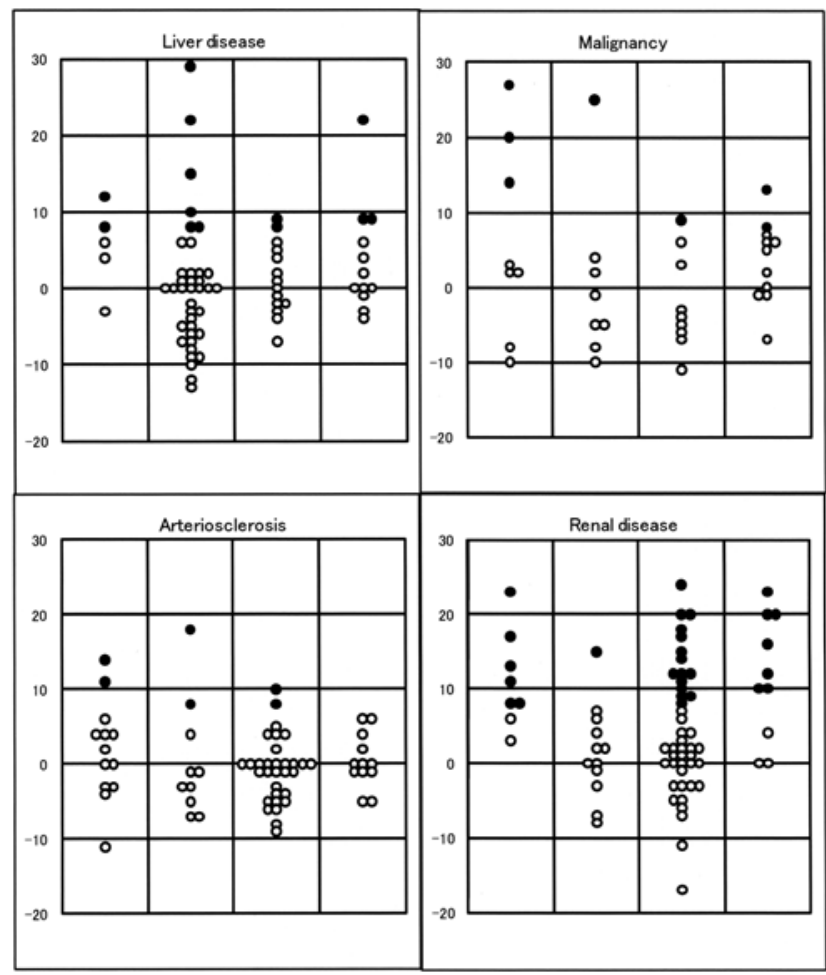

B Average CMF

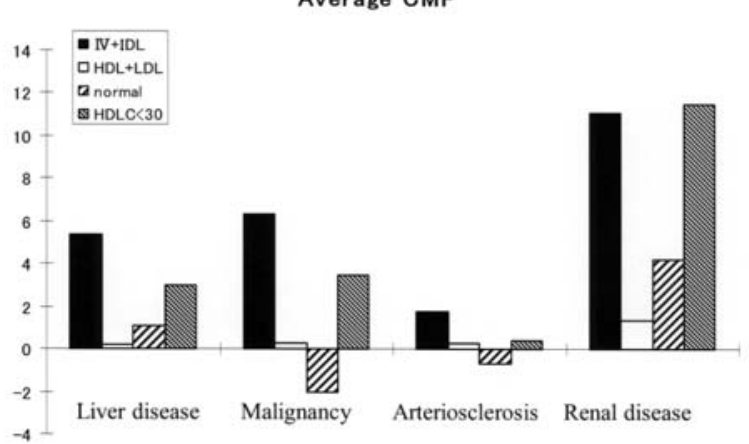

C

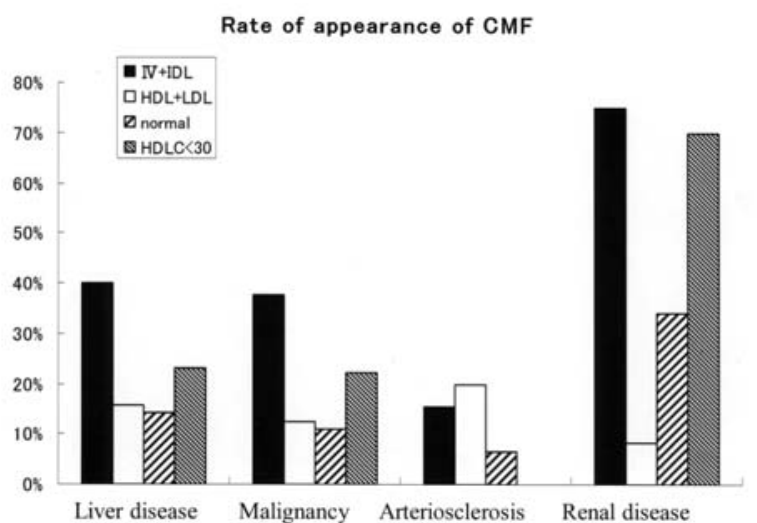

Figure 4. Distribution, mean and appearance rate of CMF in relation to lipid patterns. Scatter diagram, mean and rate of appearance of CMF according to lipid patterns. (A) Scatter diagram of CMF. (B) Mean of CMF. (C) Rate of appearance of CMF.

Mean CMF (Fig. 4B) was 5.4 in IV+IDL and 3.0 in low HDL, which are slightly high values in liver diseases and malignant tumors. In malignant tumors, mean CMF was low (-2.0) in normal type. In arteriosclerosis, mean CMF was close to 0 in all patterns, ranging from -0.7 to 1.8 . In renal diseases, mean CMF was high in all patterns excluding HDL+LDL, 4.2 in normal type, 11.1 in IV+IDL, and 11.5 in low HDL.

CMF appearance rate was also compared among lipid patterns (Fig. 4C). The findings in liver diseases and malignant tumors were similar. The appearance rate was high (38-40\%) in IV+IDL, $11-14 \%$ in normal type, $13-16 \%$ in HDL+LDL, and $22-23 \%$ in low HDL. The rate was generally low in arteriosclerosis, but the pattern of the appearance rate was opposite to the pattern in other disease groups. In renal diseases, the appearance rate was low (8\%) in HDL+LDL, but was very high in the other lipid patterns, $37 \%$ in normal type, $70 \%$ in low HDL, and $75 \%$ in IV+IDL.

\section{Discussion}

There are primary and secondary hypocholesterolemia classifications, but only secondary hypocholesterolemia is clinically useful for diagnosis and severity assessment.

Winder et al (5) investigated hypocholesterolemia in inpatients, and found that mean TC levels were significantly lower in death cases than in surviving cases, mortality rate of patients with hypocholesterolemia was $\sim 10$ times that in patients with mean levels of TC, and no patients had TC levels of $\leq 45 \mathrm{mg} / \mathrm{dl}$. Based on these findings, they suggested that TC levels are useful as a parameter of clinical prognosis. Okumura et al (6) investigated the possibility of TC screening in order to predict cause of death in Japanese patients, and found that the frequency of death from coronary arterial disease was $>3$ times than that of liver cancers in Japanese patients with hypocholesterolemia who died within 5 years, in whom the mortality rate from hyperlipidemia-associated coronary arterial disease is low. Bakalar et al (7) followed changes in TC and its precursors in patients with major traumas, and found that the cause of hypocholesterolemia was decreased TC synthesis. Concentrations of precursors (lanosterol and lathosterol) were significantly lower when compared with controls, and lathosterol levels were suggested to be useful as a marker of TC synthesis. The above reports suggest that hypocholesterolemia is an important risk factor that increases mortality in various diseases.

We considered the mechanism of hypocholesterolemia in 4 disease groups, liver diseases, malignant tumors, arteriosclerotic and renal diseases in patients with secondary hypocholesterolemia as follows. In comparison of TC in relation to disease and lipid patterns, TC levels ranged from $97-119 \mathrm{mg} / \mathrm{dl}$ in liver diseases, showing no significant differences among lipid patterns, other than $79.0 \mathrm{mg} / \mathrm{dl}$ in low HDL.

Regarding deaths within 1 year after measurement as death cases, the mortality rates of patients showing different lipid patterns were compared (Fig. 3). Within 1 year, the mortality rate of hypocholesterolemic patients was low in arteriosclerosis and renal diseases, which suggests that hypocholesterolemia is not a significant prognostic factor. In contrast, numerous hypocholesterolemic patients with liver diseases and malignant tumors died, thus suggesting that hypocholesterolemia is an important risk factor in these conditions. In liver diseases, TC and HDL-C were markedly low (TC, $15-38 \mathrm{mg} / \mathrm{dl}$; HDL-C, $3.7-12.5 \mathrm{mg} / \mathrm{dl}$ ) in 3 patients; prognosis was poor, and all 3 patients died after $\sim 1$ month. In comparison of mean TC in 
survivors and deceased patients among the disease groups and lipid patterns, mean TC was 90 and 72 , respectively, in abnormal LDL, and 95 and 43, respectively, in low HDL in liver diseases, thus confirming that $\mathrm{TC}$ is lower in death cases with these 2 lipid patterns. No consistent trends were noted for other patterns.

With regard to classification of hypocholesterolemia by lipid patterns (Fig. 1), disease specificities were noted (Fig. 2A). The highest frequency of HDL+LDL (44.7\%) was noted in liver diseases, while the frequency was $14.7-21.6 \%$ in other diseases. The frequency of abnormal LDL was also high $(12.9 \%)$ in liver diseases, but it occurred in only 1 patient $(1.5 \%)$ in the other disease groups. Normal type accounted for $59.5 \%$ of patients with renal diseases and $45.6 \%$ of patients with arteriosclerotic diseases. The highest frequency of low HDL (29.7\%) was noted in malignant tumors.

In liver diseases, lipid levels decreased with pathological progression in the order of VLDL, LDL and HDL. TC also decreased, and the appearance of HCC and TC levels at the time of death may be related. TC also decreases when liver cancer impairs overall liver function, but the direct cause of death is typically HCC. Thus, patients without HCC survived for 18 days, even though TC decreased to $15 \mathrm{mg} / \mathrm{dl}$. Based on lipid patterns, liver impairment may have been mild in patients with normal type, as the mortality rate was $0 \%$. In contrast, the mortality rate was $21.1 \%$ in HDL+LDL, in which liver impairment progressed and VLDL disappeared, and was $54.5 \%$ in abnormal LDL, which represents a more severe state with decreased HDL.

In malignant tumors other than liver cancers, the mortality rate was $22.2 \%$ in normal type and $87.5 \%$ in HDL+LDL. No abnormal LDL, which represents aggravation of liver parenchymal impairment, was noted, and this may be due to the high mortality with HDL+LDL, resulting from impairment of liver function associated with cancer metastasis to the liver, or multiple organ failure.

Clinically, secondary hypocholesterolemia most frequently occurs in liver diseases. As the liver synthesizes and secretes lipids, such as TG and ester cholesterol, lipids (particularly TC) decrease when the liver parenchyma is impaired. TG decreases less frequently, and thus, type IV cases with high TG levels are not rare. However, interferon therapy for chronic hepatitis $\mathrm{C}$ changes the lipid profile, and TG and VLDL-TG have increased according to some reports $(12,18)$.

It has been reported that cancer is more likely to develop in hypocholesterolemia, that cancer patients are more likely to develop hypocholesterolemia, and that decreases in TC and pathology are correlated (10). An increase in LDL-C uptake by tumor cells is considered to be the cause (19). Tatidis et al (20) investigated TC metabolism in patients with acute myelogenous leukemia. They measured blood concentrations of the major excreted TC, bile acid, and found a marked decrease when compared to healthy controls, showing that conversion of TC to bile acid decreased and resulted in hypocholesterolemia in patients with acute myelogenous leukemia.

Although the causes of arteriosclerosis are complex and diverse, investigation of the roles of oxidized Chol and fatty acid in dietary therapy has suggested that oxidized lipids play an important role in the progression of arteriosclerosis. Its mechanism has not been elucidated, but Staprans et al (21) reported that oxidized lipids, not TC levels, promote arteriosclerosis. In our study, 3 patients with arteriosclerosis died, but no characteristic TC levels, lipid patterns or CMF were noted.

Iseki et al (14) reported that hypocholesterolemia is an independent sign of death for chronic dialysis patients, and the survival rate after 5 years was highest in patients with a TC level of 200-219 mg/dl, followed by the group with $220 \mathrm{mg} / \mathrm{dl}$ or higher, with the survival rate being lowest in the group with TC levels $<140 \mathrm{mg} / \mathrm{dl}$. In another report (22), blood concentrations of tumor necrosis factor- $\alpha$ and IL- 6 were significantly correlated with mortality rate in hypocholesterolemic dialysis patients.

The distribution, mean and appearance rate of CMF were compared among the disease groups (Fig. 4), and differences were noted. CMF was high in many cases of renal disease, and the mean and appearance rate were highest. CMF changed after dialysis, and the findings differed depending on dialysis conditions and type of dialysis membrane. In patients with familial complex-type hyperlipidemia, lipids and CMF were compared before and after dialysis was performed 10 times between September 2004 and January 2005. Pre-dialysis TC levels were normal in 4 dialyses and high in 6 dialyses, but post-dialysis levels were normal in 2 dialyses and showed hypocholesterolemia in 8 dialyses. CMF increased after dialysis in 8 of 10 dialyses. TG markedly decreased after all 10 dialyses. Lipids decreased after dialysis, but CMF increased in most cases, thus suggesting that although lipids are removable, degenerated lipids, particularly degenerated LDL, are not removable. Therefore, the ratio of degenerated LDL may have increased due to LDL composition, and the LDL peak may have shifted toward the anode, increasing CMF.

Life-expectancy is short, and arteriosclerosis and heart diseases are likely to occur and progress in dialysis patients, and the involvement of oxidized LDL and advanced glycation end products (AGE) is considered to be the cause $(23,24)$. Itabe et al (23) measured blood oxidized LDL in dialysis patients using a monoclonal antibody, and found that it was higher than that in the control group. However, in our study, oxidized LDL and CMF were not correlated in individual dialysis patients. CMF may not have been correlated with a single risk factor, oxidized LDL, because the mobility of CMF is based on changes in the charge affected by all degenerated LDL forms, including oxidized LDL, AGE and glycated LDL.

Sampathkumar et al (24) reported that formation of high concentrations of AGE is an important mechanism in hyperglycemia-associated vascular complications. AGE is correlated with microangiopathy in diabetes, and AGE levels increase in the order of cases with no complications, retinopathy, and retinopathy + nephropathy. Accumulation of AGE in circulation in a state of renal failure may promote active oxygen- and oxidative stress-degenerated protein production, thereby impairing the vascular wall.

Lipid patterns in renal diseases are very characteristic; many cases showed normal type and high CMF. Although these are not directly related to progression of the pathology in the short term, this pattern may be the most useful CMF-combined risk factor in the long-term follow-up of renal diseases.

Hypocholesterolemia has been a risk factor for various diseases in numerous reported cases. Addition of lipid pattern 
classification and CMF to analysis software may increase clinical usefulness.

\section{References}

1. Kido T, Kurata H, Matsumoto A, Tobiyama R, Musha T, Hayashi K, Tamai S, Utsunomiya K, Tajima N, Fidge N, Itakura H and Kondo K: Lipoprotein Analysis using agaroae gel electrophoresis and differential staining of lipid. J Atherscler Thrombo 8: 7-13, 2001.

2. Sparks DL and Phillips MC: Quantitative measurement of lipoprotein surface charge by agarose gel electrophoresis. J Lipid Res 33: 123-130, 1992.

3. Harris T, Feldman JJ, Kleinman JC, Ettinger WH Jr, Makuc DM and Schatzkin AG: The low cholesterol-mortality association in a national cohort. J Clin Epdemiol 465: 595-601, 1992.

4. Jacob D, Blackburn H, Higgins M, Reed D, Iso H, McMillian G, Neaton J, Nelson J, Potter J, Rifkind B, Rossour J, Shekelle R and Yusuf S: Report of the Conference on Low Blood Cholesterol: Mortality associations. Circulation 86: 1046-1060, 1992.

5. Winter E, Ewers-Grabow U, Thiery J, Walli A, Seidel D and Greten H: The prognostic value of hypocholesterolemia in hospitalized patients. Clin Investig 72: 939-943, 1994.

6. Okumura T, Kadowaki T, Hayakawa T, Kita Y, Okayama A, Ueshima $\mathrm{H}$ and Nippon Data80 Reseach Group: What cause of mortality can we predict by cholesterol screening in the Japanese general population? Intern Med 253: 169-180, 2003.

7. Bakalar B, Hyspler R, Pachl J and Zadak Z: Changes in cholesterol and its precursors during the first days after major trauma. Wien Klin Wochenschr 115: 775-779, 2003.

8. Nguyen K, Sigaudy S and PhilipN: MCA/MR syndrome with hypocholesterolemia related to familial dominant hypobetalipoproteinemia. Am J Med Genet A 121: 109-112, 2003.

9. Brooks-Wilson A, Marcil M, Clee SM, Zhang LH, Roomp K, van Dam M, Yu L, Brewer C, Collins JA, Molhuizen HO, Loubeer O, Ouclette BF, Fichter K, Ashbourne-Exoffon JK, Sensen CW, Scherer S, Mott S, Martindake D, Frohlich J, Morgan K, Koop B, Pimstone S, Kasteleiin JJ, Genset J Jr and Haydon MR: Mutation in ABC1 in Tngier disease and familial high density lipoprotein deficiency. Nat Genet 22: 336-345, 1999.

10. Kawakami M: Secondary hypocholesterolemia. Nippn Rinsho 59: 266-270, 2001.

11. Fernandez-Miranda C, Castellano G, Guijarro C, Fernandeez I, Schoebel N, Larumbe S, Gomez-Izquierdo T and del Palacio A: Lipoprotein changes in patients with chronic hepatitis $\mathrm{C}$ treated with interferon- $\alpha$. Am J Gastroenterol 93: 1901-1904, 1998.

12. Siemianowicz K, Gminski J, Stajszczyk M, Wojakowski W, Goss M, Machalski M, Telega A, Brulinski K and Magiera-Molendpwsska H: Serum total cholesterol and triglyceride levels in patients with lung cancer. Int Mol Med 5: 201-205, 2000.
13. Iseki K, Yamazato M, Tozawa M and Takishita S: Hypocholesterolemia is a significant predictor of death in a cohort of chronic hemodialysis patients. Kidney Int 61: 1887-1893, 2002.

14. Atac B, Brahaji D, Frishman WH and Lerner R: Anemia and hypocholesterolemia. Heart Dis 5: 65-71, 2003.

15. Bonville DA, Parker TS, Levine DM, Gordon BR, Hydo LJ, Eachempati SR and Barie PS: The relationships of hypocholesterolemia to cytokine concentrations and mortality in critically ill patients with systemic inflammatory response syndrome. Surg Infect 5: 39-49, 2004.

16. Dunham CM, Fealk MH and Sever WE 3rd: Following severe injury, hypocholesterolemia improves with convalescence but persists with organ failure or onset of infection. Crit Care 7: 145-153, 2003.

17. Naeem M, Bacon BR, Mistry B, Britton RS and Di Bisceglie AM: Changes in serum lipoprotein profile during interferon therapy in chronic hepatitis C. Am J Gastroenterol 96: 2468-2472, 2001 .

18. Nakagawa T, Ueyama Y, Nozaki S, Yamashita S, Menju M, Funahashi T, Kameda-Takamura K, Kubo M, Tokunaga K and Tanaka T: Marked hypocholesterolemia in a case with adrenal adenoma-enhanced catabolism of low density lipolrotein (LDL) via the LDL receptors of tumor cells. J Clin Endocrinol Metab 80: 92-96, 1995.

19. Tatidis L, Vitols S, Gruber A, Paul C and Axelson M: Cholesterol catabolism in patients with acute myelogenous leukemia and hypocholesterolemia: suppressed levels of a circulatingmarker for bile acid synthesis. Cancer Lett 170: 169-175, 2001.

20. Itabe H, Yamamoto $H$, Imanaka T, Shimamura K, Uchiyama $H$, Kimura J, Sanaka T, Hata Y and Takano T: Sensitive detection of oxidatively modified low density lipoprotein using a monoclonal antibody. J Lipid Res 37: 45-53, 1996.

21. Staprans I, Pan XM, Rapp JH and Feingold KR: The role of dietary oxidized cholesterol and oxidized fatty acids in the development of atherosclerosis. Mol Nutr Food Res 49: 1075-1082, 2005.

22. Chiang CK, Ho TI, Hsu SP, Peng YS, Pai MF, Yang SY, Hung KY and Tsai TJ: Low-density lipoprotein cholesterol: association with mortality and hospitalization in hemodialysis patients. Blood Purif 23: 134-140, 2005.

23. Bologa RM, Levine DM, Parker TS, Cheigh JS, Serur D, Stenzel KH and Rubin AL: Interleukin-6 predicts hypoalalbuminemia, hypocholesterolemia, and mortality in hemodialysis patients. Am J Kidney Dis 32: 107-114, 1998.

24. Obialo CI, Okonofua EC, Nzerue MC, Tayade AS and Riley LJ: Role of hypoalalbuminemia and hypocholesterolemia as copredictors of mortality in acute renal failure. Kidney Int 56: 1058-1063, 1999.

25. Sampathkumar R, Balasubramanyam M, Rema M, Premanand C and Mohan V: A novel advanced glycation index and its association with diabetes and microangiopathy. Metabolism 54: 1002-1007, 2005. 